assessment of incontinence although should probably not have been on a cancer pathway, 2 had a previous failed colonoscopy and 1 had learning difficulties. There was a single cancer in this patient group. Despite having a reasonable justification for exclusion from a STT examination 27/70(39\%) patients still went on to have an endoscopic examination. We found no justification for exclusion from a STT examination in $34 / 104(33 \%)$ patients of whom $31(91 \%)$ went on to have a colonoscopy following a clinic review and two of these patients had a colorectal cancer.

Conclusion Two-thirds of patients judged inappropriate for a STT examination were appropriately identified by primary care referrers. However, there was no justification for why a third of patients were excluded and the large majority of these proceeded to a colonoscopy. This data supports secondary care providers being the key decision makers in allocation of patients for $2 \mathrm{ww}$ STT colonoscopy examinations.

\section{PTH-19 ASSESSING THE IMPACT OF SBAR PROFORMA AND GI BLEED ROTA IN MANAGING VARICEAL BLEED PATIENTS}

${ }^{1}$ Irfan Ullah* ${ }^{2}$ Amir Hazizi Abdul Razak, ${ }^{3}$ Gireesh Kothegal Marimahadevappa, ${ }^{4}$ Peter Neville. ${ }^{1}$ Nevill Hall Hospital, Abergavenny, UK; ${ }^{2}$ Prince Charles Hospital, Merthyr Tydfil, UK; ${ }^{3}$ Prince Charles Hospital, Merthyr Tydfil, UK; ${ }^{4}$ Prince Charles Hospital, Merthyr Tydfil, UK

\subsection{6/gutjnl-2021-BSG.334}

Introduction Variceal bleeding in liver disease is associated with significant mortality and morbidity. Early recognition and appropriate interventions are crucial in managing patients with acute variceal bleeding.

Methods Patients presented with acute variceal haemorrhage to Prince Charles Hospital and Royal Glamorgan Hospital were retrospectively identified using our endoscopy database. Four locally agreed key performance factors including early endoscopy within 24 hours, use of prophylactic antibiotics, documentation of Glasgow Blatchford Score (GBS) and correct identification of upper gastrointestinal bleed (UGIB) on initial presentation were assessed in patients presented between February 2018 to February 2019 in the initial audit cycle.These factors were audited again for patients from March 2019 to December 2019, after the introduction of SBAR proforma and out-of-hours (OOH) GI bleed on call rota.

Results A total of 31 patients were included in initial audit and 13 patients in the reaudit.In the first audit it showed that compliance with endoscopy within 24 hours was 96\%, prophylactic antibiotics was 74\%, GBS documentation was 55\% and identification of upper GI Bleed on admission was $100 \%$. After the introduction of UGI bleed SBAR proforma and $\mathrm{OOH}$ GI bleed on call rota, the repeat audit of the same factors showed improvement in GBS documentation to $92 \%$ and prophylactic antibiotics use on admission to $85 \%$ while UGIB identification on admission remained 100\%. Endoscopy within 24 hrs of admission was $85 \%$, but this was due to the risk benefit review of a case and the need for urgent endoscopy by the team.Endoscopic procedures done $\mathrm{OOH}$ initially was $22 \%$ which rose to $31 \%$ after introduction of UGI bleed on call service. Additional information also showed that SBAR proforma was used in $62 \%$ of cases. It also showed better use of the in hours endoscopy services, as more cases were discussed appropriately with the on call Gastroenterologists.

Conclusion This audit concludes that there was better documentation of GBS, use of prophylactic antibiotics, appropriate early recognition and management of variceal bleeding by using available endoscopy service and the appropriate use of on call service for these patients, who generally have significant morbidity due to their underlying liver disease. We recommended that repeating the audit cycle in 12-24 months would be valuable to see whether these improvements are sustained and to reassess the long term benefit of GI bleed on call service in effectively managing these patients. We also suggest that we should aim to achieve 100\% compliance in these performance factors along with appropriate management of associated liver disease.

\section{PTH-20 THE IMPACT OF COVID-19 PANDEMIC ON UPPER GI AND HEPATO-PANCREATO-BILIARY CANCERS - DIAGNOSIS AND STAGING}

${ }^{1,2}$ Ee Xuan Ngeyu* ${ }^{*}$ Palaniappan Jeyam-Suresh, ${ }^{1,3}$ Anjan Dhar. ${ }^{1}$ County Durham and Darlington Nhs Foundation Trust, Darlington, UK; ${ }^{2}$ Newcastle university Medical School, Newcastle, UK; ${ }^{3}$ School of Health and Life Sciences, Teesside University, Middlesbrough, UK

\subsection{6/gutjnl-2021-BSG.335}

Introduction Cancer Charities and the BSG Endoscopic Section predict an increased GI cancer related mortality arising from reduced endoscopic activity and delays to cancer diagnosis during the COVID-19 pandemic. Initial modelling reports suggest up to a $58 \%$ reduction in weekly GI cancer detection. A real world analysis of the adverse impact of COVID-19 on Upper GI and hepato-pancreato-biliary (HPB) cancers has not been reported so far.

Aims and Methods Patients with a new diagnosis of upper GI (oesophageal and gastric) or HPB cancer at County Durham over two 4-month periods of June-September 2019 (preCOVID) and 2020 (peri-COVID) were selected for this analysis. Data collected from electronic patient notes and Somerset Cancer MDT electronic database included: 1) patient demographics and co-morbidities; 2) Pathway parameters - duration of symptoms prior to presentation, 2WW timeframes, interval between referral and endoscopy, performance status, elective vs emergency presentation, and 3) Tumour parameters - cancer site, TNM staging. 90-day mortality, where available was also recorded.

Results 163 patients were included - 82 patients in preCOVID (2019) and 81 in peri-COVID (2020) cohorts. In the pre-COVID group, 25 were oesophageal cancers, 8 gastric, 21 pancreatic, and 26 liver cancers. In the peri-COVID group, 31 were oesophageal cancers, 13 gastric, 17 pancreatic, and 18 liver cancers. An absolute increase of $17.5 \%$ was seen in emergency presentation of UGI cancers to A\&E in 2020 $(35.8 \% \mathrm{v} 18.3 \%)$. In the peri-COVID group, $87.1 \%$ of patients had advanced (TNM staging of T3/4, N2 or M1) oesophageal and gastric cancer, $7.1 \%$ more than in preCOVID group. A similar increase of $6.4 \%$ was identified in metastatic pancreatic cancers $(58.8 \%$ in $2020 \mathrm{v} 52.4 \%$ in 2019). There was a significant reduction in the number of endoscopies performed within 3 weeks of referral - 56.8\% pre-COVID, compared with only $20 \%$ peri-COVID. There was no significant difference between 60-day mortality (preCOVID 25.61\% and peri-COVID group 25.91\%) and 90-day mortality $(34.15 \%$ and $32.10 \%$ respectively), between the two groups.

Conclusion Although the impact of COVID-19 on endoscopy activity resulted in a $17.5 \%$ increase in emergency presentations of UGI and HPB cancers and nearly $10 \%$ increase in 